\title{
An Examination and Categorical Analysis of Squamous Cell Carcinoma
}

\author{
Tamara Golosarsky ${ }^{1}$ and Lisa LaRochelle ${ }^{1 \#}$ \\ ${ }^{1}$ Bishop Guertin High School, Nashua, NH, USA \\ \#Advisor
}

ABSTRACT

This article discusses the compository nature and hierarchical metastatic proliferation of Squamous Cell Carcinoma. Discussion ranges from the effects of the disease from the cellular level all the way to entirety of the body, including the impact of UV radiation and mutation on cells such as melanocytes and congruent epithelia. Current and potential future cures for the disease, such as immunotherapy and Mohs' Surgery are explained in application to the disease, along with the history behind each practice and how it came into use by physicians. Lastly, hopes for effective recognition and continued research of squamous cell carcinoma are laid out, stressing the importance of directing attention to the second most common skin cancer in the medical-research community.

\section{Introduction}

Skin cancers are extremely serious diseases that can readily impact and change a patient's life. Particular skin cancers are more deadly than others depending on a variety of factors such as: tendency to metastasize, location, growth rate, specific types of cells involved, etc. These stated factors divide up the broad category of skin cancer into three types: squamous cell carcinoma, basal cell carcinoma, and melanoma. The first type listed, squamous cell carcinoma, is a cancer that is categorized by an abnormal, rapid growth of squamous cells, and is considered to be the second most common type of skin cancer. Notably, squamous cell carcinoma is not just limited to the skin. This cancer may also develop the lungs, mouth, throat and esophagus (Baptist Health, n.d.). Within this work, attention will be focused upon the intricacies and complications of skin-based squamous cell carcinoma. Stages of the disease, systematic description at various levels of organization of the human body, treatment and recovery, will also be discussed in thorough format and methodology. Formulation of total information will lead to a hopeful outlook on the future of squamous cell carcinoma, postulated and assiduous in nature.

\section{Review of Literature}

Twenty-five pieces of literature were assessed for this literary review, including a variety of journal papers, hospital website databases and clinical websites. Most of these resources were found through databases such as PubMed, NCBI and PMC. Information provided from these collected resources focused on five key topics: the population most affected by squamous cell carcinoma, risk factors that increase chances of developing squamous cell carcinoma, proliferation and metastasis of squamous cell carcinoma, the history of the cancer and, the current treatment used to battle the disease. Each topic was addressed through either theoretical proposition and testing, known statistics, proven fact, or historical analysis. Throughout this range of topics and approaches regarding squamous cell carcinoma, every present piece of literature provided thorough, idiosyncratic insight into the disease. 


\section{High Risk Factors and Population Affected}

Primarily, it is of utmost importance to discuss the general pool of patients that is plagued by squamous cell carcinoma. Nearly anyone may be stricken with the disease. Simply put, it is not a cancer that discriminates. However, there are various factors that increase the risk of exposure. Those with lighter skin, meaning less production of the protective pigment melanin, are more likely to have their DNA exposed to harmful UV rays. Mutation may occur Personal histories of skin cancer and/or precancerous lesions may also contribute to pre-established genetic factors that indicate an increased likelihood of developing squamous cell carcinoma. For example, patients with Xeroderma pigmentosum cannot repair damage caused by UV light/radiation, opening a greater possibility that cells may turn cancerous (American Academy of Dermatology, n.d.). Even those with inborn or medically induced weaker immune systems may also be at higher risk (Mayo Clinic, 2019). A stunted reactionary immune response can lead to debilitated, defensive epidermal dendritic cells and therefore, a development of a tumor. Lack of specific lymphocytes called B cells and $\mathrm{T}$ cells, which aid in development of antibodies and recognition of foreign pathogens, may also allow cancerous growth to spread (Cancer Research UK, 2014). Any prolonged and excessive exposure to the sun, artificial or otherwise, can also cause vast disruption of squamous cell DNA. Potential mutation in DNA could lead to extreme, cancerous growth of said squamous cells (Mayo Clinic, 2019). All of these prominent risk factors contribute to the one million cases of squamous cell carcinoma that are diagnosed a year, roughly equating to 115 cases per hour. Other statistics show that, in the past three decades, squamous cell carcinoma diagnoses have increased by 200 percent (The Skin Cancer Foundation, 2020c).

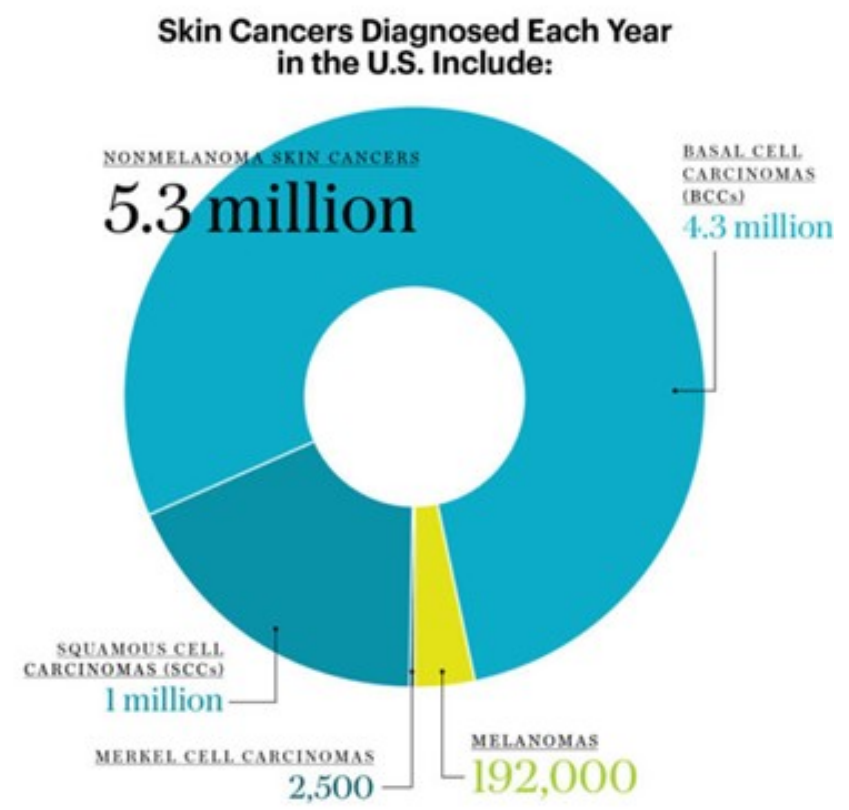

Figure 1. Breakdown of skin cancer diagnoses based on type of cancer

As indicated by Figure 1, one million people diagnosed with squamous cell carcinoma each year. Out of those diagnosed, 15,000 people die from SCC within the United States alone (The Skin Cancer Foundation, 2020b). Clearly, from the data described, squamous cell carcinoma spreads rapidly and affects the daily lives of people globally. Even with such prevalence of diagnosis however, the discovery of squamous cell carcinoma within the body is usually surprising for those affected. SCC's stealthy spread lends no external symptoms, besides the occasional formation of a small red nodule or a minute flaky ulceration (Mayo Clinic, 2019). To make sense of the commonality of 
aforementioned diagnosis, understanding how squamous cell carcinoma proliferates is vital to assessing the causal effects of the disease.

\section{Squamous Cell Carcinoma Proliferation: From Cell to Body}

Typically, squamous cell carcinoma is not considered by dermatologists to be life-threatening; if the disease pervades unchecked, however, deterioration of the lymphatic system and key organs may pose a greater risk to health, and even the possibility of death. The cancer takes hold beginning inside the resident cells of the epidermal layer of the skin, specifically the stratum spinosum layer (SEER Training, n.d.). A healthy stratum spinosum contains newly formed epithelium, which mitotically originate the stratum basale layer, the deepest layer of the epidermis. These cells are continually pushed up into more superficial compact layers, which form the overall stratification of the cutaneous membrane. In a patient with squamous cell carcinoma, the DNA of squamous cells that constitute the stratum spinosum has mutated, producing ineffective signaling/coordination of cellular growth. The most common cause of squamous cell DNA disruption is overexposure to the sun's UV radiation, specifically the UVB type (Firnhaber, 2012, p. 161). Under normal circumstances, messenger RNA copies lines of nucleotide sequencing, subsequently ordering its host cell to construct functional proteins coded within given DNA. In irradiated DNA, crucial hydrogen bonds that connect nucleotides together are broken, causing coding errors in the form of nucleotide gaps in said sequencing (Rastogi et al., 2010, p. 7). Thus, messenger RNA copies these errors and transmits instruction to its host cell. Due to innate flaw of instruction, faulty proteins are produced, creating unchecked cellular growth. Transmission of erred code is only exacerbated by intense, repeated overexposure to the sun, which can lead to sunburn and therefore, inflammation of the skin. This explicit inflammatory response occurs as local epithelium regenerate in order to replenish ineffective and damaged epithelium (Boness, 2012). As more cells become produced to compensate for perceived damage, the error in DNA replicates within the cells, creating rapidly proliferating cancer.

Thus, as new cells move up from the stratum basale, they become crowded among the unchecked cancerous squamous cells, rather than dividing normally and partaking in sequential progression of superficial movement through the epidermis. This is known as Bowen's disease, the earliest form of squamous cell carcinoma, which can also be referred to as squamous carcinoma in situ. Visually, Bowen's disease appears as a small patch of red, scaly skin that can potentially be raised (Morton, 2017). Since it is referred to as squamous cell carcinoma in situ, Bowen's disease is only superficial and has not yet spread to other tissues. The cancerous cells begin to produce an oversupply of keratin, a water-resistant lipoprotein, stiffening both the stratum spinosum and stratum corneum (Punovuori $\&$ Wickström, 2020, p. 355). In effect, the rapidly producing cancer is trapped and therefore, forced to move deeper into the epidermis.

Theories have been proposed as to how the cancer spreads beyond the deeper layers of the skin. The most prominent hypothesis involves the migratory carcinogenic cells creating strain upon the underlying epidermal basement membrane as they expand. These pressured cancerous exertions are hypothesized to be the explanation of how the growing, malignant cells spread to both the dermis and the subcutaneous membrane, because since the cancerous cells have limited room to expand, they are compelled to break away from the original tumor. Consequently, the cells rupture the basement membrane barrier and invade the lower dermis. Stratification becomes disrupted and unorganized, lacking the clear layer divisions seen in stainings of uninfected individuals. The common functional connection of epidermal ridge papillae is also vastly affected by the breakdown of the epidermal basement membrane (Punovuori \& Wickström, 2020, p. 355). As they continue to move deeper into the dermis, cancerous squamous cells come into contact with nerve endings and prominent root vasculatures/plexuses. In-transit metastasis occurs through these tissue planes as independent malignant cells can enter both local lymph node nodules and blood vessels, which ultimately connect to the bloodstream and overall lymphatic system (National Cancer Institute, 2017). For example, if the primary origin of a patient's SCC is the buccal region, then the cancer would metastasize by entering the neighboring blood vessels and into the regional cervical node. Other common nodes that are centers for transmission of 
distant metastasis include the axillary, parotid and inguinal lymph nodes (Transplant Skin Cancer Network, n.d.). Through these variants of fluid connective tissue, the cancer can efficiently travel to other healthy tissues and organs. Normally, nutrients and waste are collected through lymph and blood-based circulation, allowing function of tissues and overall organs is reinforced. However, as the cancer follows the established path of nutrient-rich propagation, it uses fluid connective tissue's disseminative properties to take hold within surrounding organs. Cancerous SCC cells begin to rapidly divide further, causing inflammatory responses of visceral/neighboring organ cells and even the host organ itself. Virulent cellule infiltration induces a protective reaction by immune cells known as neutrophils (Cancer Research UK, 2014). At the molecular level, neutrophils begin to formulate the enzymes myeloperoxidase (MPO) and elastase (ELA), which would typically aid in defensive attack against foreign pathogens. In the cause of SCC or even any cancer in general, these enzymes erode natural extracellular barriers. A particular type of ELA, called neutrophil elastase, is very effective at breaking down the fibronectin, proteoglycans, and type IV collagen that constitute many of the protective cellular membranes that make up organs within the body (Gasparoto et al., 2012, p. 46). Membrane deterioration allows the cancerous cells to spread even further, as histological barriers become ineffective in keeping the cancer in one place. Tumors form, creating misshapen and lumpy superficial surfaces and functionality begins to fail as established structure deteriorates.

The positive feedback loop of caustic, malignant SCC cells motivates a continual apportionment of the disease, leading to possible organ and organ system metastasis of the lungs, bone, central nervous system, and liver (Transplant Skin Cancer Network, n.d.). Taking a look at the lungs, the general pattern of organ system and subsequently, total bodily metastasis, is clear: once the cancer has deteriorated the pleural lining, hindering both absorption and filtering qualities that healthy simple squamous epithelia provide at this location, the cancer is able to move to the neighboring trachea and diaphragm tissue. The process only repeats itself, leaving no functional support or available toxin filtration and nutrient permeability that would be seen in a healthy system. Therefore, the metastatic SCC cells move farther into surrounding systematic connections, regardless of initial location of metastasis.

If the cancer reaches total bodily metastasis, also known as stage 4 cancer, prognosis typically dictates that multiple organ systems have been and will effectively be overrun by multiplying SCC tumor cells. Prognosis at this time would indicate that the chance of survival is relatively low (Watson, 2020). Normally, interconnected functionalities of organs and overall organ systems can no longer be performed due to the multitude of metastatic tumors in the body (Cancer Research UK, 2017). Cancerous inhabitation in the spine, lungs, liver, brain and skull hinders communication of the CNS and responsiveness of the brain, forcing a lack of ability to maintain homeostasis, and thus, bodily shutdown. Lack of regulation in one's internal environment inevitably indicates that death will most likely occur. Yet, depending on personal medical history, specific location of metastasis and size and growth of the tumors, SCC does not have to get to this point. Developed over a fairly recent timeline, new and inventive procedures have been put into place to effectively combat SCC and help patients deal with the shock of their diagnosis.

\section{History and Current Treatment}

The prospect and diagnosis of skin cancer has only lately become a focus for many dermatologists. Bowen's disease itself was only identified and discovered in 1912, by its namesake, Dr. John T. Bowen (Kaldas, 2020). From this discovery, successive experiments by many other dermatologists and scientists began to be performed in order to discover the potential causes of skin cancer. Results attained in the totality of the 19th and 20th centuries pointed to UV radiation being the sole cause, with epithelial pigmentation aiding in prevention of nucleic acid damage. In the 1970s, the discovery of specific suppressive tumor effectors, found within the DNA of familial individuals, aided in the understanding of how hereditary history affected diagnosis of SCC and skin cancer in general. Aforementioned treatment for SCC advanced alongside the sequential discoveries and applications detailed above. Shifting from the simple localized removal of tumors that was popularized in the 16th century, a technique known as Mohs surgery arose during the 1930s. Pioneered by Dr. Frederic E. Mohs, Mohs surgery attended to a patient's cancer in stages ("The History of Skin Cancer," 2015). First, a dermatologist would identify the location of the tumor, and then, very 
carefully, remove a thin layer of cancerous surface tissue using a scalpel. Next, the sample would be traced to provide a map of total surface area affliction within the patient. Lastly, the tissue sample would be frozen and stained to highlight edges and underlying portions of the excision. If the physician noted the presence of cancerous cells at these locations, another removal of skin would be performed (The Skin Cancer Foundation, 2020a). In the 1940s-1950s, surgery developed into a chemical-based, direct elimination of rapidly developing cells in the form of chemotherapy. Chemotherapy is not used as a typical treatment of SCC currently, but depending on location and overall spread of metastasis, it can be considered as an effective treatment (Moffitt Cancer Center, 2018). Immunotherapy, on the other hand, is definitively the most effective treatment available today. This treatment trains a patient's immune system to better recognize cancerous cells and attack them when they become prevalent. Success of immunotherapy comes from its ability to tailor immunity defense mechanisms directly to the individual environment of the patient, rather than a simple attack on all rapidly multiplying cells within the body (National Cancer Institute, 2019b). In 2018, a drug called Libtayo was approved by the FDA for SCC treatment. It was the first successful immunotherapy to be applied to squamous cell tumors (National Cancer Institute, 2019a). More developments in the field of immunology and oncology are helping patients battle with metastatic SCC, making this development certainly not the last.

\section{Methods}

The methodology behind curating the literature present within this literature review was centered upon both the reliability and prevalence of information provided. Initially, a pool of fifty resources was collected from biomedical databases based on their immediate coverage of squamous cell carcinoma and how informative this coverage was. The next stage of assessment of literature focused on the prevalence, thoroughness and repetition of information presented on squamous cell carcinoma. Out of the pool that was collected, thirty-six pieces of literature maintained unique, detailed information about the cancer.

Next, reliability of the resources was assessed on three different fronts: 1) the institution from which the resource came, 2) the currency of the resource's publication, and 3) whether the resource was edited or peer-reviewed before publication. When addressing the first criterion, well-known institutions such as Nature carried more credibility and were more readily considered for the information that they provided. The second criterion limited timing of publishing to the contemporary, ranging from the 2010s to the current day in order to eliminate outdated findings. Lastly, the third criterion eliminated the use of untrustworthy information to guarantee that the information provided within the literature review is correct and suitable for the public. Through the entire stage of literary assessment, twenty-five pieces of literature were chosen as the final candidates that were utilized within this literature review of squamous cell carcinoma.

\section{Discussion}

As a result of conducting research that utilizes literature studying the topic of squamous cell carcinoma as its main focal point, it becomes clear that the entirety of the disease is not completely understood. Many aspects of the cancer continue to remain a mystery, including both definitive proliferation and metastasis, why certain groups of people are affected more than others and how the cancer develops to begin with (Punovuori \& Wickström, 2020, p. 355). Various hypotheses and theories, embodied by the literature studied within the current literature analysis, have been put forward regarding addressing the truths behind these elusive characteristics of squamous cell carcinoma, but none have conclusively been proved. Thus, present research does not address these attributes of the disease completely, indicating that there is a greater need for more research to be conducted upon these distinct topics in relation to squamous cell carcinoma. 


\section{Conclusions and Implications}

Squamous cell carcinoma is a vastly intricate cancer. It can manifest itself in several ways and affect a wide scale of the population based on various genetic and environmental factors. Today, there is a wide variety of treatments that can be undertaken, depending on the stage and condition of the cancerous tumor that is being treated. However, due to the absence of well-defined research regarding the fundamental properties of squamous cell carcinoma, many diagnosed with the later stages of the cancer may have missed immediate and intensive care.

The ramification of uncomprehending key principles of squamous cell carcinoma is that of a battle between life and death. If a greater comprehension of the disease is sought after, those who have been diagnosed with the metastatic stage of the cancer will have a greater fighting chance at life. This is because squamous cell carcinoma is inconsequential if discovered and treated early (Mayo Clinic, 2019). With a larger understanding of cancer formation, proliferation, and metastasis, squamous cell carcinoma will be identified faster, allowing for prevention of further tumor growth. Furthermore, dermatologists equipped with thorough knowledge of the cancer can easily monitor and follow up with tumors, improving patient recovery drastically as a result (Firnhaber, 2012, p.167). Thus, cultivating a greater scientific focus on squamous cell carcinoma would result in improvement of the patient survival rate and allow many more people to live healthy and content lives to their fullest extent.

\section{Future Research/Treatment}

Techniques such as reflectance confocal microscopy (RCM) and optical coherence tomography (OCT) are now being used to assess lesions without their physical removal. Research into why certain SCCs spread more than others is also being conducted. A protein present in certain SCC cases known as INPP5A has been linked to rate of metastasis within patients. Specifically, scientists have linked lower levels of this protein to a higher chance of spreading within the body. Lastly, treatments such as the use of retinoids, Nicotinamide (a form of vitamin B3) and "targeted drugs" or pharmaceuticals that directly tailor themselves to the composition of a patient's SCC, are currently undergoing trials. Effectiveness and patient pool vary from treatment to treatment, offering a wide range of possibilities for future prescribed medication (American Cancer Society, 2020). With ever-improving technologies, established treatments, and dissection of tumor proliferation, squamous cell carcinoma will develop from a mysterious ailment, to a known and understood cancer, proven to be easily treatable and non-consequential in everyday life.

\section{Limitations}

Due to the scarcity of extensive and dedicated research to solely squamous cell carcinoma, there are still many variables regarding proliferation, metastasis, and origination within the body. In the past, studies concerning skin cancer have mainly focused on melanoma, the deadliest skin cancer that is diagnosable today. Unfortunately, squamous cell carcinoma has not gotten the same academic attention as melanoma, thus making it vastly unresearched. Furthermore, because there is little known about the disease, literature concerning squamous cell carcinoma and its proliferation is purely theoretical. This does not provide a succinct or accurate assessment of the disease's growth and how that growth corresponds to patient symptomology. On the other hand, literature that does contain proven facts about squamous cell carcinoma tends to be repetitive and does not expand upon this known information. Since the conducted literature review is based only on the described available literature, these limitations may create research an incomplete analysis of squamous cell carcinoma.

To remedy the stifling need for both research and literature on squamous cell carcinoma, time is required for interest and technology to work together in order to yield more succinct research into the disease. Advancing medical technologies and persistent researchers have made it is very likely that future research will bring about clearer 
conclusions of the basis of how squamous cell carcinoma multiplies within the body and how symptoms of the disease relate to this multiplication.

\section{Acknowledgements}

The author would like to thank Lisa LaRochelle for providing the learning environment which piqued her curiosity into researching squamous cell carcinoma. Concurrently, the author thanks Bishop Guertin High School as an institution for providing the resources and space for research to be thoroughly conducted. Lastly, the author thanks both Boris Golosarsky and Nora Kirsh for their unyielding support and dedication to the author's pursuit of researching squamous.

\section{References}

American Academy of Dermatology. (n.d.). Skin cancer types: Squamous cell carcinoma causes. Retrieved November 9, 2020, from https://www.aad.org/public/diseases/skin-cancer/causes

American Cancer Society. (2020, July 8). Basal and squamous cell skin cancer research | latest skin cancer research. Retrieved November 9, 2020, from http://www.cancer.org/cancer/basal-and-squamous-cell-skin-cancer/about/new-research.html

Bain, J., \& Kamowitz, B. (2019). Early detection starts with you, part 1 [Pie Chart]. The Skin Cancer Foundation. Retrieved November 7, 2020, from https://www.skincancer.org/blog/early-detection-starts-with-you/

Baptist Health. (n.d.). Squamous cell skin carcinoma: Causes and treatment. Retrieved November 3, 2020, from https://www.baptisthealth.com/services/cancer-care/types-of-cancer/squamous-cell-carcinoma

Boness, L. (2012, July 10). What happens when we get sunburnt? Science Illustrated. Retrieved November 8, 2020 , from https://www.scienceillustrated.com.au/blog/science/what-happens-when-we-get-sunburnt/

Cancer Research UK. (2014, October 29). The immune system and cancer. Retrieved November 8, 2020, from https://www.cancerresearchuk.org/about-cancer/what-is-cancer/body-systems-and-cancer/the-immune-system-andcancer

Cancer Research UK. (2017, July 26). Stages and grades of skin cancer. Cancer Research Uk. Retrieved November 8, 2020, from https://www.cancerresearchuk.org/about-cancer/skin-cancer/stages-grades

Firnhaber, J. M. (2012). Diagnosis and treatment of Basal cell and squamous cell carcinoma. American Family Physician, 86(2), 161-168. Retrieved November 6, 2020, from http://www.aafp.org/afp/2012/0715/p161.html

Gasparoto, T. H., de Oliveria, C. E., de Freitas, L. T., Pinheiro, C. R., Ramos, R. N., da Silva, A. L., Garlet, G. P., da Silva, J. S., \& Campanelli, A. P. (2012). Inflammatory events during murine squamous cell carcinoma development. Journal of Inflammation, 9(1), 46. Retrieved November 7, 2020, from https://doi.org/10.1186/1476-9255-9-46

Kaldas, M. V. (2020, March 22). Bowen Disease. Medscape. Retrieved November 8, 2020, from https://www.emedicine.medscape.com/article/1100113-overview 
Mayo Clinic. (2019, June 21). Squamous Cell Carcinoma of the Skin. Mayo Foundation for Medical Education and Research. Retrieved November 6, 2020, from https://www.mayoclinic.org/diseases-conditions/squamous-cell-carcinoma/symptoms-causes/syc Moffitt Cancer Center. (2018). Squamous Cell Carcinoma Chemotherapy. Retrieved November 8, 2020, from https://www.moffitt.org/cancers/squamous-cell-carcinoma/treatment/chemotherapy/

Morton, C. A. (2017, January 3). Bowen Disease. NORD (National Organization for Rare Disorders). Retrieved November 6, 2020, from https://www.rarediseases.org/rare-diseases/bowen-diseasewww.rarediseases.org/rare-diseases/bowen-disease/

National Cancer Institute. (2017, February 2). Metastatic cancer: When cancer spreads. Retrieved November 6, 2020, from https://www.cancer.gov/types/metastatic-cancer

National Cancer Institute. (2019a, June 4). Advances in melanoma and other skin cancers research. Retrieved November 8,2020 , from https://www.cancer.gov/types/skin/research

National Cancer Institute. (2019b, September 24). Immunotherapy for cancer. Retrieved November 8, 2020, from https://www.cancer.gov/about-cancer/treatment/types/immunotherapy

Punovuori, K., \& Wickström, S. A. (2020). How cancer invasion takes shape. Nature, 585(7825), 355-356. Retrieved November 7, 2020, from https://doi.org/10.1038/d41586-020-02490-3

Rastogi, R. P., Richa, Kumar, A., Tyagi, M. B., \& Sinha, R. P. (2010). Molecular mechanisms of ultraviolet radiation-induced dna damage and repair. Journal of Nucleic Acids, 2010, 1-32. Retrieved November 6, 2020, from https://doi.org/10.4061/2010/592980

SEER Training. (n.d.). Layers of the skin. National Cancer Institute. Retrieved November 5, 2020, from https://www.training.seer.cancer.gov/melanoma/anatomy/layers.html

The history of skin cancer. (2015). Journal of the American Academy of Dermatology, 72(5), AB27. Retrieved November 8, 2020, from https://doi.org/10.1016/j.jaad.2015.02.121

The Skin Cancer Foundation. (2020a, September 17). Mohs surgery. Retrieved November 8, 2020, from https://www.skincancer.org/treatment-resources/mohs-surgery/

The Skin Cancer Foundation. (2020b, September 17). Skin cancer facts \& statistics. Retrieved November 3, 2020, from https://www.skincancer.org/skin-cancer-information/skin-cancer-facts/

The Skin Cancer Foundation. (2020c, October 2). Squamous cell carcinoma. Retrieved November 8, 2020, from https://www.skincancer.org/skin-cancer-information/squamous-cell-carcinoma/

Transplant Skin Cancer Network. (n.d.). Metastatic Squamous Cell Carcinoma. Retrieved November 7, 2020, from https://www.skincancer.ucsf.edu/metastatic-squamous-cell-carcinoma

Watson, S. (2020, February 19). Stage 4 squamous cell carcinoma: Prognosis and outlook. Healthline. Retrieved November 8, 2020, from https://www.healthline.com/health/stage-4-squamous-cell-carcinoma-prognosis-and-outlook 